\title{
Etiology and an Integrated Management of Severe Hemoptysis Due to Pulmonary Tuberculosis
}

\author{
Song Yang*\#, Zhuanying Mai, Xiangzhen Zheng, Yueling Qiu* \\ Department of Pulmonary Medicine, The $175^{\text {th }}$ Hospital of People's Liberation Army, Affiliated Dongnan \\ Hospital of Xia'men University, Zhangzhou City, China \\ Email: \#ir596yangsongq@aliyun.com, maizhuanying@aliyun.com, zhengxiangzhen@aliyun.com, \\ qiuyaoling@aliyun.com
}

Received 30 December 2014; accepted 27 January 2015; published 3 February 2015

Copyright (C) 2015 by authors and Scientific Research Publishing Inc.

This work is licensed under the Creative Commons Attribution International License (CC BY). http://creativecommons.org/licenses/by/4.0/

(c) (i) Open Access

\section{Abstract}

Background: It is very important to enhance the therapeutic effect and prognosis of severe tuberculous hemoptysis after the determining of its etiological cause and the source of bleeding. The etiology and integrated curative effect of severe hemoptysis due to pulmonary tuberculosis among 112 inpatients were analyzed. Materials and Methods: The cause was retrospectively analysed. The integrated management effect after the follow-up of mean three years in 112 cases with severe hemoptysis being resulted from pulmonary tuberculosis from June 2008 to July 2012 was described. Active pulmonary tuberculosis ranked the first cause of lower respiratory tract bleeding $(32 / 112,28.5 \%)$, followed by old pulmonary tuberculosis $(28 / 112,25.0 \%)$, tuberculous bronchiectasis $(25 / 112,22.3 \%)$, purified tuberculous cavity $(12 / 112,10.7 \%)$, fungal infection in old pulmonary tuberculosis cavity $(9 / 112,7.1 \%)$, or broncholithiasis $(6 / 112,5.4 \%)$. Almost all suffers with severe hemoptysis were treated by an integrated management, including psychology, anticoagulants, vasoconstrictor agents. Etiological treatment including anti-tuberculosis and anti-infection was simultaneously or subsequently involved. Sixty-four inpatients with severe hemoptysis being failed to be cured by medical treatment were then received selective bronchial artery embolization. Four patients were received surgical wedge resection, lobectomy or pneumonectomy. The total cure rate added up to $\mathbf{9 8 . 2 \%}$ after mean three years' follow-up. The mortality was $1.8 \%$. Conclusions: Active pulmonary tuberculosis was still responsible for the severe hemoptysis in the southeast region of China. Severe hemoptysis of pulmonary tuberculosis was also resulted from stable tuberculosis, tuberculous bronchiectasis, tuberculosis cavity, fungal infection, or broncholithiasis. Better clinical therapeutic effect could be attained by early etiological diagnosis and comprehensive treatment strategy.

\footnotetext{
*These authors contributed equally to this work.

${ }^{\#}$ Corresponding author.
} 


\section{Keywords}

\section{Severe Hemoptysis, Pulmonary Tuberculosis, Medical Treatment, Selective Bronchial Artery Embolization, Lobectomy, Pneumonectomy}

\section{Introduction}

Hemoptysis is a relatively common presenting acute respiratory symptom in patients seen by pulmonary specialists [1]. Especially severe hemoptysis may be fatal, which requires emergent and appropriate treatment. It may present as a life-threatening symptom with a mortality rate of up to $50 \%-100 \%$ in the absence of adequate and prompt management [2] [3]. Severe hemoptysis includes major or massive hemoptysis. The definition of major and massive haemoptysis may vary in the literatures. Expectoration of 200 to $600 \mathrm{ml}$ of blood in a day is defined as "major" hemoptysis, while the amount above this level is generally accepted as "massive" hemoptysis [4] [5]. Severe hemoptysis constitutes $1 \%-1.5 \%$ of all hemoptysis cases and can be life threatening either as a result of compromised gas exchange or because of circulatory collapse secondary to acute blood loss [6].

Undoutedly, successful treatment of severe hemoptysis depends on a rapid ascertainment of the pathogeny. Consequently an early diagnosis of the etiology of severe hemoptysis can be of importance. It not only helps to improve the cure rate, but also reduce complications and lower its mortality. About the cause of severe hemoptysis, geographic distribution may differ accordingly [7]. Its etiology varies from different disorders, which need different treatment option and have distinct prognosis accordingly. Particularly, massive hemoptysis should be needed urgent internal, interventional, or surgery treatment, otherwise it might be life-threatening medical event. Some patients can be treated successfully with endobronchial interventions. Selective bronchial artery embolization (BAE) can be rewarding in some patients but the recurrence rate is higher in tuberculosis than other etiologies of severe hemoptysis. Up to now, surgical resection of the lung, mainly lobectomy, remains an extremely important measure for survival. But it should be performed very selectively to avoid higher postoperative morbidity and mortality [5]. In our retrospective study, the etiology and curative effect of severe hemoptysis among 112 tuberculous inpatients were analyzed. The assessment of long-term therapeutic effect was explored after three years' follow-up. Before our clinical observational study started, this investigation was approved by our hospital Ethics Committee.

\section{Materials and Methods}

\subsection{Diagnosis}

The inpatients with severe hemoptysis were consecutive and enrolled in our study from the outpatient department and the department of emergency. All inpatients were clinically diagnosed by the following tests, including the analysis of peripheral veinal blood cell, seral prothrombin, C-reactive protein, purified protein derivative test, acid-fast bacilli of sputum smear, bacterial culture of sputum, sputum culture of mycobacterium tuberculosis, seral anti-tuberculous antibody test, chest radiologic examinations (X-ray, high resolution computed tomography). Meanwhile flexible fiberoptic bronchoscopy, percutaneous lung puncture biopsy guided by type B color ultrasound were performed. Severe hemoptysis included major and massive bleeding from lower respiratory tract. Diagnostic criteria for the volume of severe hemoptysis are more than $200 \mathrm{ml}$ of blood in a day [5]. Active pulmonary tuberculosis almost manifested afternoon fever, night sweat, cough, expectoration, hemoptysis, generl tiredness, loss of weight, or anorexia. The existing standard diagnostic approach of active pulmonary tuberculosis required performing three initial sputum smear examinations [8]. For those whose smear is negative, the diagnosis is based on a chest X-ray, a trial of broad-spectrum antibiotics and clinical judgment.

\subsection{Treatment}

Firstly, all inpatients were received psychological guide and were advised not to be nervous. They were received repeated exhortations to lie absolutely quiet in bed for preventing from asphyxia and respiratory tract obstruction. Simultaneously, they were undergone immediate conservative medical treatment including reversal of anticoa- 
gulants, vasoconstrictor agents of small pulmonary arteries (for example pituitrin), vasodilator drugs of peripheral veins or blood capillary, or other hemostatic drugs. For these patients complicating with hypertension or mental stress, whose hemoptysis was extraordinary difficult to be controlled within short-term. Phentolamine, sodium nitroprusside, nitroglycerin and some other antihypertensive drugs were administered to control blood pressure around 90/60 mmHg for the benefit of rapid heal of hemorrhagic foci. This method also could create conditions for the treatment of primary disease.

Etiological treatment was also taken simultaneously or subsequently. Etiologic therapy was also involved by the use of anti-tuberculosis drugs (isoniazid, rifampicin, ethambutol, pyrazinamide) for active pulmonary tuberculosis, antibiotics for tuberculous bronchiectasis with infection or lung fungal infection. If they failed to respond to medical treatment, then these invalids were further coped with flexible fiberoptic bronchoscopy, or interventional treatment by selective bronchial artery embolization. Under local anesthesia, the common femoral artery was percutaneously punctured, and a $5 \mathrm{~F}$ introduction sheath was inserted. A flush catheter was advanced into the upper part of the descending thoracic aorta, and a diagnostic anteroposterior angiogram was performed, which in all these cases with severe hemoptysis cases revealed the hypertrophic bronchial arteries. The hypertrophic bronchial arteries were then selectively catheterized with a $5 \mathrm{~F}$ cobra-shaped curved catheter. Transcatheter embolization of the hypertrophic bronchial arteries subsequently performed through the catheter after stabilization of the catheter tip was confirmed. A microcatheter was used if there was opacification of the important spinal branches. Tris-acryl gelatin microspheres 500 - $700 \mu \mathrm{m}$ in diameter were used as the embolization materials, and were injected slowly through $1 \mathrm{ml}$ syringes. The embolic particles were dispersed in contrast medium to allow visualization of any backflow and to monitor for progressive slowing of flow. Throughout the procedure, regular angiograms were performed to detect previously invisible connections to side branches supplying the spinal cord. Embolization was terminated when the antegrade flow ceased.

Unfortunately, if all the above-mentioned first aid treatments declared unsuccessful, prompt surgical section including wedge resection, lobectomy or pneumonectomy were ultimately adopted. Before urgent surgery, the bleeding site should be found by flexible fiberoptic bronchoscopy at operating room or bedside. After general anesthesia, single-lung ventilation was established through a double-lumen endotracheal tube. A posterolateral thoracotomy was performed.

\subsection{Follow-Up and Assessment of Curative Effect}

Fully recovered cases should be followed up for about three years outside the hospital so as to evaluate the curative effect. The statistical methods were made with the constituent ration of each etiological cause of severe hemoptyusis, the mortality percentage and the curative rate.

\section{Results}

From June 2008 to July 2012, 112 inpatients with severe hemoptysis due to pulmonary tuberculosis (72 males, 40 females; mean age, 42 years; range, 16 to 88 years) were consecutively enrolled in this study. The number of patients with different age under 20 years, between 20 to 40 years, between 40 to 60 years, over 60 years were 6 (5.4\%), 32 (28.5\%), 45 (40.2\%) and 29 (25.9\%), respectively.

Among 112 invalids with severe hemoptysis due to pulmonary tuberculosis, active tuberculosis ranked the first cause of lower respiratory tract bleeding (32/112, 28.5\%), followed by old tuberculosis (28/112, 25.0\%), tuberculous bronchiectasis (25/112, 22.3\%), tuberculosis purified cavity (12/112, 10.7\%), fungal infection in old tuberculosis cavity (9/112, 7.1\%), or broncholithiasis (6/112, 5.4\%) (Table 1).

Table 1. The constituent ration of different etiological causes suffered from severe hemoptysis due to pulmonary tuberculosis from June 2008 to July 2012.

\begin{tabular}{cccccccc}
\hline & Classification & $\begin{array}{c}\text { Active } \\
\text { tuberculosis }\end{array}$ & $\begin{array}{c}\text { Old tuberculosis } \\
\text { tuberculous } \\
\text { bronchiectasis }\end{array}$ & $\begin{array}{c}\text { Purified } \\
\text { tuberculosis } \\
\text { cavity }\end{array}$ & $\begin{array}{c}\text { Fungal infection in } \\
\text { tuberculous cavity }\end{array}$ & Broncholithiasis & Total \\
\hline $\begin{array}{c}\text { Numbers of } \\
\text { invalids }\end{array}$ & 32 & 28 & 25 & 12 & 9 & 6 & 112 \\
$\begin{array}{c}\text { Constituent } \\
\text { ration (\%) }\end{array}$ & 28.5 & 25.0 & 22.3 & 10.7 & 7.1 & 5.4 & 100 \\
\hline
\end{tabular}


All suffers with severe hemoptysis were treated by an integrated management, including psychology, anticoagulants, vasoconstrictor agents. Etiological treatment including anti-tuberculosis and anti-infection was simultaneously or subsequently involved. Sixty-four inpatients with severe hemoptysis being failed to be cured by medical treatment were then received selective bronchial artery embolization (BAE) (Figure 1, Figure 2). After BAE, they expectorated a minor amount of blood or bloody sputum, which gradually disappeared after 48 to 72 hours. No severe complications were observed in any of the cases as a result of this intervention except for moderate prothorax pain in 6 cases. But the transient chest pain disappeared within 24 hours by the oral anal-

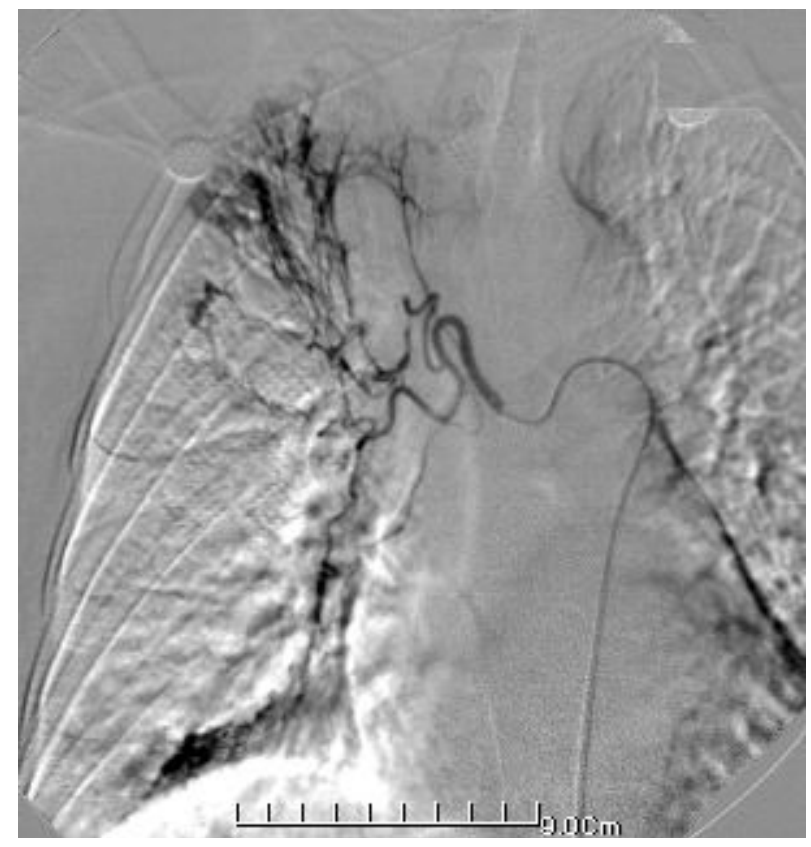

Figure 1. Radiograph showing enlarged bleeding bronchial arteries.

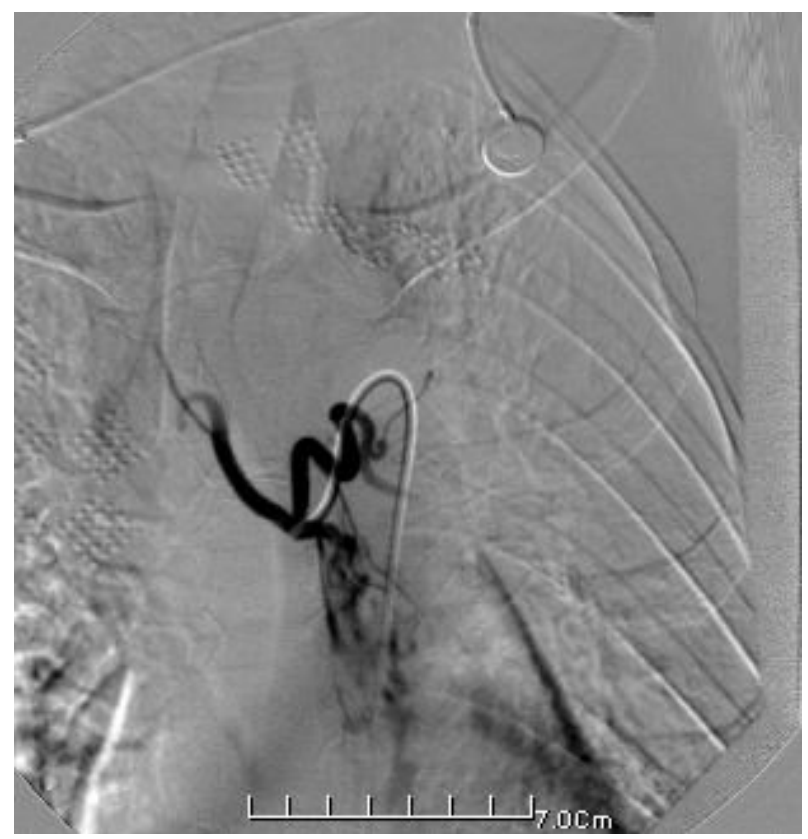

Figure 2. Radiography showing the peripheral cut off in flow after embolism. 
gesic drug treatment. After the first embolization treatment, all patients had to be undergone follow-up for mean three years (range, 24 to 76 months). 62 cases with massive hemoptysis no longer exhibited recurrent hemoptysis. But four months after the discharge, there were 2 patients with massive hemoptysis with recurrent pulmonary tuberculosis showed rebleeding. One patient with bilateral destroyed lung suffered from severe tuberculous bronchiectasis and one patient with fungal infection experienced recurrent bleeding after two years. All the four rebleeding cases were received second BAE. Among 112 patients with massive hemoptysis, four cases whose bleeding couldn't be controlled by medical and BAE treatment were alternately had to be received surgical wedge resection, lobectomy or pneumonectomy (Figures 3-5).

Among 112 severe hemoptysis, only two cases during the conservative medical treatment died from asphyxia. The mortality was $1.8 \%(2 / 112)$. The total cure rate added up to $98.2 \%(110 / 112)$ after mean three years' follow-up. The cause of death accounted for hemorrhagic shock and respiratory tract suffocation.

\section{Discussion}

The definition of hemoptysis was expectorated blood arising from the pulmonary parenchyma or tracheobron

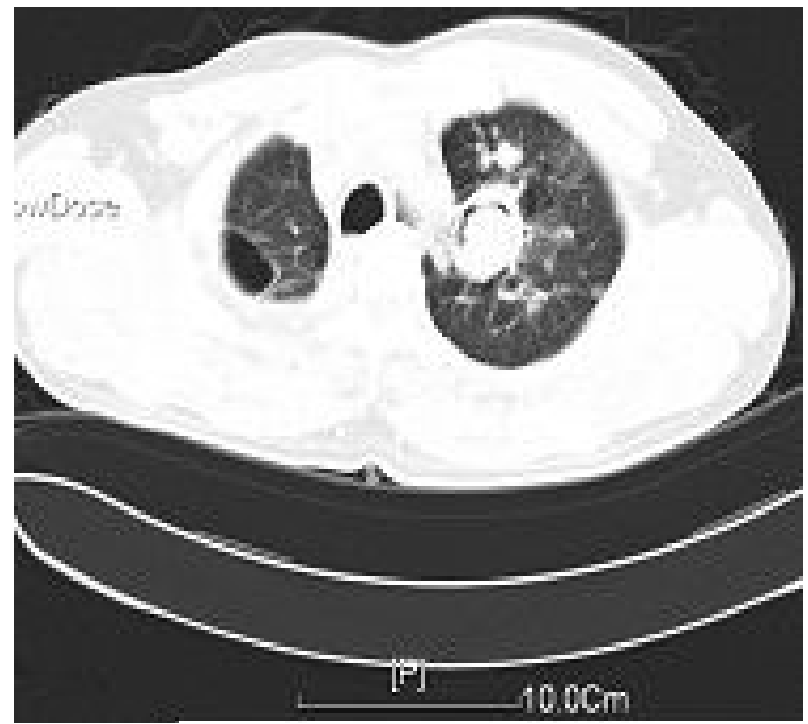

Figure 3. Chest computed tomography aspergillus revealing lesft upper tuberculosis necrotic cavity with a large aspergillus ball.

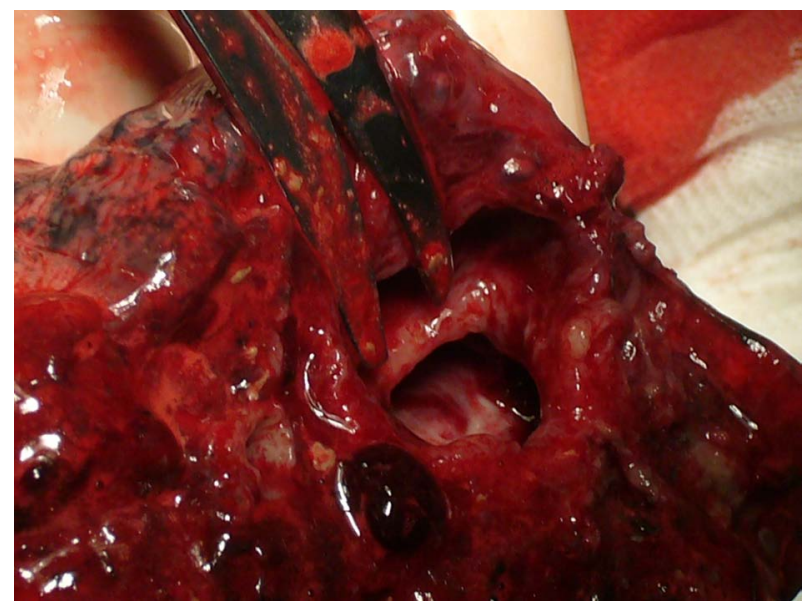

Figure 4. Postoperation of lobectomy observing two giant cavities with active bleeding in left upper lobe. 


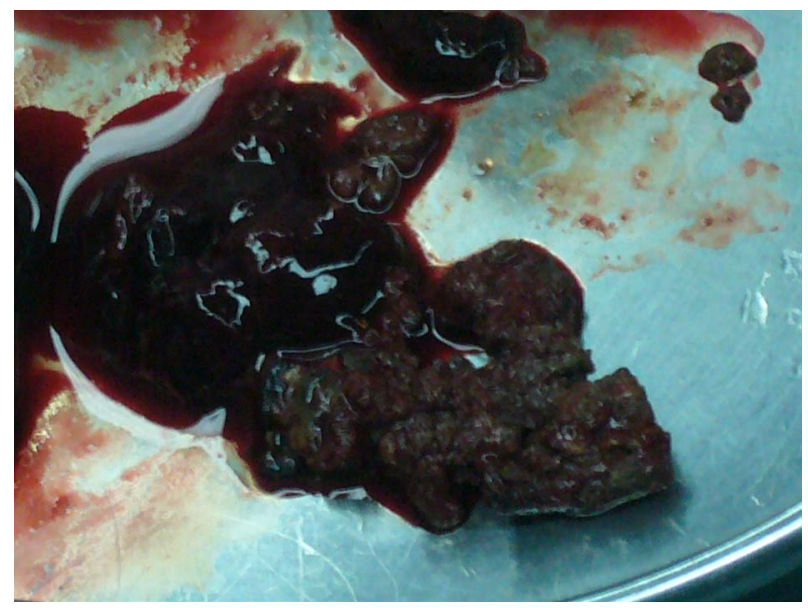

Figure 5. Postoperative ball and blood-stained tissue.

chial trees. Ninety percent of significant hemoptysis come from bronchial artery origin. Mssive hemoptysis was a life-threatening condition with a high mortality when treated conservatively. Medical treatment of massive hemoptysis carried a mortality rate of $50 \%$ to $100 \%$ [1]. Interestingly, varying definitions in the literature exist of massive hemoptysis. These range from 100 to over $1000 \mathrm{ml}$ in a 24-hour period [9].

Severe hemoptysis still be the endangerous to the health. Severe hemoptysis was a manifestation of pulmonary or tracheobronchial disease [10]. As far as we have known, 100 kinds of diseases can lead to hemoptysis [11]. Different treatment strategies were determined by the etiology of severe hemoptysis. Therefore, elucidation of the causes of severe hemoptysis should be crucial to the cure of the primary disease. It was caused by chronic lung diseases such as pulmonary tuberculosis, chronic bronchitis, bronchiectasis, lung cancer, aspergillosis, and pneumo-coniosis. But the causes of severe hemoptysis varied from different countries or regions [5]. In the developing world, the most common etiology of hemoptysis was suffered from tuberculosis [12]. But In the developed world, the most common etiologies were primary lung neoplasm [13]. Malignant airway tumors, bronchitis, and bronchiectasis are typically the most common cause of massive hemoptysis, but tuberculosis and lung abscesses among others have been reported [14]. Tuberculosis remains an important cause of hemoptysis in the United States despite the decreased prevalence compared to more endemic countries [15].

Severe hemoptysis could be lethal, when the large number of blood obstructed the trachea or bronchi. The suffocation and acute respiratory failure soon occurred. There was also a literature reported that among the various causes of hemoptysis, tuberculosis ranked the first (29.4\%), followed by lung cancer (22.7\%), and chronic bronchitis (16.2\%) [16]. In our report, the most common etiological cause of severe hemoptysis was attributable to higher morbidity of active pulmonary tuberculosis at the southern region of Fujian province in China. This was consistent with the fact that China ranked the second among the countries with high burden of tuberculosis. Tuberculosis remained a major public health concern in China, which caused 150,000 deaths per year [17]. Severe hemoptysis due to pulmonary tuberculosis in inactive stage might be associated with mechanical stretch injury of fibrous scar, secondary tuberculous bronchiectasis or bacterial infection in the purified tuberculous cavities [5].

Integrated treatment was performed in this study, which included psychological guidance, absolutely rest in bed, exercise reduction, avoiding superheating beverages or spicy food, quitting drinking alcohol and smoking. Hemostatic drugs were administered accordingly. For those without contraindications such as hypertension, pituitrin was prescribed to constrict afferent bronchopulmonary arterioles. Whereas for elderly patients with hypertension or mental stress, whose massive hemoptysis was very difficult to be controlled except for their blood pressure being decreased in the normal range. Accordingly, phentolamine, sodium nitroprusside, nitroglycerin and some other antihypertensive drugs were taken to control artery blood pressure around 90/60 mmHg.

During the treatment of severe hemoptysis, we endeavored to seek for the etiological cause of severe hemoptysis. Patents with active pulmonary tuberculosis were received anti-tuberculous treatment. The cases with bacterial lung infection were managed with antibiotics. Even though our clinicians had taken active interal medical treatment measures, sixty-four inpatients with massive hemoptysis were failed to be cured. Alternatively, they 
were then accepted emergent selective bronchial artery embolization. Bronchial artery embolization played an important role in the urgent hemostasis of severe hemoptysis [1] [11] [18]. Although there were four patients occurred to recurrent bleeding, they were cured by second embolization. BAE was very effective for obtaining immediate bleeding control in hemoptysis associated with active TB or post-TB sequelae. It was important to observe whether or not rebleeding occurs up to 1 year of BAE especially in TB patients with aspergilloma. Even rebleeding can be managed well by second BAE [19].

Although few severe complications were observed in our clinical study, it was very important to observe and reduce the complications of BAE. More recent series report few complications, which is likely due to improvement in contrast mate-rials and technique. Rare cases of paraplegia, transient paraplegia, and transient BrownSéquard syndrome have been reported following bronchial artery embolizations, with an overall risk of transverse myelitis of less than $1 \%$. Postembolization syndrome, consisting of retrosternal chest pain, inter-costal pain, transient dysphagia, and fever is not uncommon. Other reported complications include stroke, esophageal ulceration, bronchial infarction and stenosis, bronchoesophageal fistula, transient left orbital and forehead pain, and transient neuro-logical symptoms [1] [20].

Four patients were received surgical resection. After about three years' follow-up, the patients with surgical section no longer showed rebleeding. Once the site of bleeding in patients with massive hemoptysis has been localized, pulmonary resection offers the patient the best chance of survival. The operation was involved in wedge resection, lobectomy or pneumonectomy. Management of massive hemoptysis and timing of surgical intervention pose difficult problems. Gourin and Garzon have recommended prompt surgical resection for patients having more than $600 \mathrm{~mL}$ blood in 24 hours. For such a patient mortality rate is $18 \%$ by surgery as compared to 75\% rate in those treated conservatively [21]. Emergency surgery should be reserved only for those patients: 1) having adequate lung function; 2) exact site of bleeding definitely defined; 3) continuing bleeding despite the adequate measures taken [22]. Reported operative mortality was approximately $17 \%$ as compared to the $50 \%$ 70\% mortality from non-surgical management [23]. In our report, two lethal massive hemoptysis cases suffered airway obstruction and suffocation. The mortality was 1.8\% (2/112). Preventing suffocation and aspiration resulted from massive hemoptysis was essential to reduce the mortality. The total cure rate added up to $98.2 \%$ (110/112) after mean three years' follow-up.

\section{Conclusion}

In brief, active pulmonary tuberculosis was responsible for the severe hemoptysis in the southeast region of China, followed by old tubefculosis and tuberculous bronchiectasis. To the greatest extent, the clinical therapeutic effect could be acquired by precise etiological diagnosis and comprehensive treatment strategy involved in psychological, mecdical, BAE, or resectional surgery. We should reduce the complications of BAE and accurately assess the timing of surgical intervention management of massive hemoptysis. The mortality should be reduced by preventing from asphyxia and suffocation. Better clinical therapeutic effect had been attained by early etiological diagnosis and comprehensive treatment strategy, but few limitations in our study were existed such as limited sample size, short follow-up period. So the assessment of the total therapeutic effect by comprehensive treatment with large sample size and a longer time should be needed for further study.

\section{Competing Interests}

The authors declare that they have no financial or non-financial competing interests.

\section{References}

[1] Sidhu, M., Wieseler, K., Burdick, T.R. and Shaw, D.W.W. (2008) Bronchial Artery Embolization for Hemoptysis. Seminars in Interventional Radiology, 25, 310-318. http://dx.doi.org/10.1055/s-0028-1085931

[2] Najarian, K.E. and Morris, C.S. (1998) Arterial Embolization in the Chest. Journal of Thoracic Imaging, 13, 93-104. http://dx.doi.org/10.1097/00005382-199804000-00004

[3] Conlan, A.A., Hurwitz, S.S. and Krige, L. (1983) Massive Hemoptysis. Review of 123 Cases. The Journal of Thoracic and Cardiovascular Surgery, 85, 120-124.

[4] Winter, S.M. and Ingbar, D.H. (1988) Massive Hemoptysis: Pathogenesis and Management. Journal of Intensive Care Medicine, 3, 171-188. http://dx.doi.org/10.1177/088506668800300306

[5] Halezeroğlu, S. and Okur, E. (2014) Thoracic Surgery for Haemoptysis in the Contexts the Best Management Ap- 
proach? Journal of Thoracic Disease, 6, 182-185.

[6] Jean-Baptiste, E. (2000) Clinical Assessment and Management of Massive Haemoptysis. Critical Care Medicine, 28, 1642-1647. http://dx.doi.org/10.1097/00003246-200005000-00066

[7] Syabbalo, N. (1991) Hemoptysis: The Third-World Perspective. Chest, 99, 1316-1317. http://dx.doi.org/10.1378/chest.99.5.1316b

[8] Lin, H.H., Dowdy, D., Dye, C., Murray, M. and Cohen, T. (2012) The Impact of New Tuberculosis Diagnostics on Transmission: Why Context Matters. Bulletin of the World Health Organization, 90, 739A-747A. http://dx.doi.org/10.2471/BLT.11.101436

[9] Lordan, J.L., Gascoigne, A. and Corris, P.A. (2003) The Pulmonary Physician in Critical Care Illustrative Case 7: Assessment and Management of Massive Haemoptysis. Thorax, 58, 814-819. http://dx.doi.org/10.1136/thorax.58.9.814

[10] Hahn, S., Kim, Y.J., Kwon, W., Cha, S.W. and Li, W.Y. (2010) Comparison of the Effectiveness of Embolic Agents for Bronchial Artery Embolization: Gelfoam versus Polyvinyl Alcohol. Korean Journal of Radiology, 11, 542-546. http://dx.doi.org/10.3348/kjr.2010.11.5.542

[11] Zhou, Z.H. (2009) Hemoptysis-Pathogenesis, Diagnosis and Treatment Progress. Modern Preventive Medicine, 36, 1398-1400.

[12] Rémy, J., Arnaud, A., Fardou, H., Giraud, R. and Voisin, C. (1977) Treatment of Hemoptysis by Embolization of Bronchial Arteries. Radiology, 122, 33-37. http://dx.doi.org/10.1148/122.1.33

[13] Yoon, W., Kim, J., Kim, Y., Chung, T. and Kang, H. (2002) Bronchial and Nonbronchial Systemic Artery Embolization for Life-Threatening Hemoptysis: A Comprehensive Review. Radiographics, 22, 1395-1409. http://dx.doi.org/10.1148/rg.226015180

[14] Hirchberg, B., Biran, I., Glazer, M. and Kramer, M.R. (1997) Hemoptysis: Etiology, Evaluation, and Outcome in a Tertiary Referral Hospital. Chest, 112, 440-444. http://dx.doi.org/10.1378/chest.112.2.440

[15] McGuinnes, G., Beacher, J.R., Harkin, T.J., Garay, S.M., Rom, W.N. and Naidich, D.P. (1994) Hemoptysis: Prospective High-Resolution CT/Bronchoscopic Correlation. Chest, 105, 1155-1162. http://dx.doi.org/10.1378/chest.105.4.1155

[16] WHO (2002) WHO Report 2002. Global Tuberculosis Control. Surveillance, Planning, Financing. World Health Organization, Geneva.

[17] Li, Y.Y. (1997) Etiology Analysis on Hemoptysis in 520 Elderly Patients. Nanjing Railway Medical College Journal, 16, 189-190.

[18] Parrish, S., Krimsky, W., Browning, R. and Alabrash, M. (2012) Novel Approaches to the Patient with Massive Hemoptysis. Journal of Community Hospital Internal Medicine Perspectives, 2, 14784-14787. http://dx.doi.org/10.3402/jchimp.v2i1.14784

[19] Hwang, H.G., Lee, H.S., Choi, J.S., Seo, K.H., Kim, Y.H. and Na, J.O. (2013) Risk Factors Influencing Rebleeding after Bronchial Artery Embolization on the Management of Hemoptysis Associated with Pulmonary Tuberculosis. Tuberculosis and Respiratory Diseases, 74, 111-119. http://dx.doi.org/10.4046/trd.2013.74.3.111

[20] Corr, P.D. (2005) Bronchial Artery Embolization for Life-Threatening Hemoptysis Using Tris-Acryl Microspheres: Short-Term Result. CardioVascular and Interventional Radiology, 28, 439-441. http://dx.doi.org/10.1007/s00270-004-0227-x

[21] Gourin, A. and Garzon, A.A. (1974) Operative Treatment of Massive Hemoptysis. The Annals of Thoracic Surgery, 18, 52-60. http://dx.doi.org/10.1016/S0003-4975(10)65717-7

[22] Erdogan, A., Yegin, A., Gürses, G., et al. (2005) Surgical Management of Tuberculosis-Related Hemoptysis. The Annals of Thoracic Surgery, 79, 299-302. http://dx.doi.org/10.1016/j.athoracsur.2004.05.016

[23] William, A.B. and James, B.D.M. (1989) Recurrent Major Haemoptysis: Progression to Pneumonectomy. Thorax, 35, 905-906. 
Scientific Research Publishing (SCIRP) is one of the largest Open Access journal publishers. It is currently publishing more than 200 open access, online, peer-reviewed journals covering a wide range of academic disciplines. SCIRP serves the worldwide academic communities and contributes to the progress and application of science with its publication.

Other selected journals from SCIRP are listed as below. Submit your manuscript to us via either submit@scirp.org or Online Submission Portal.
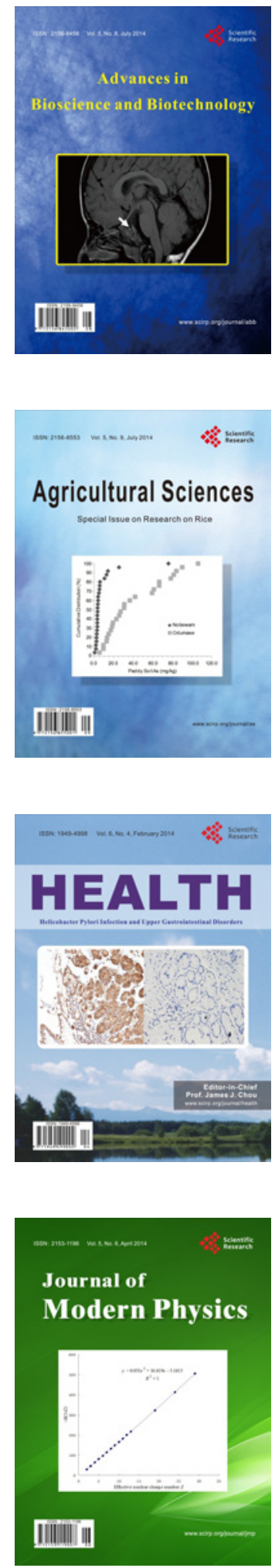
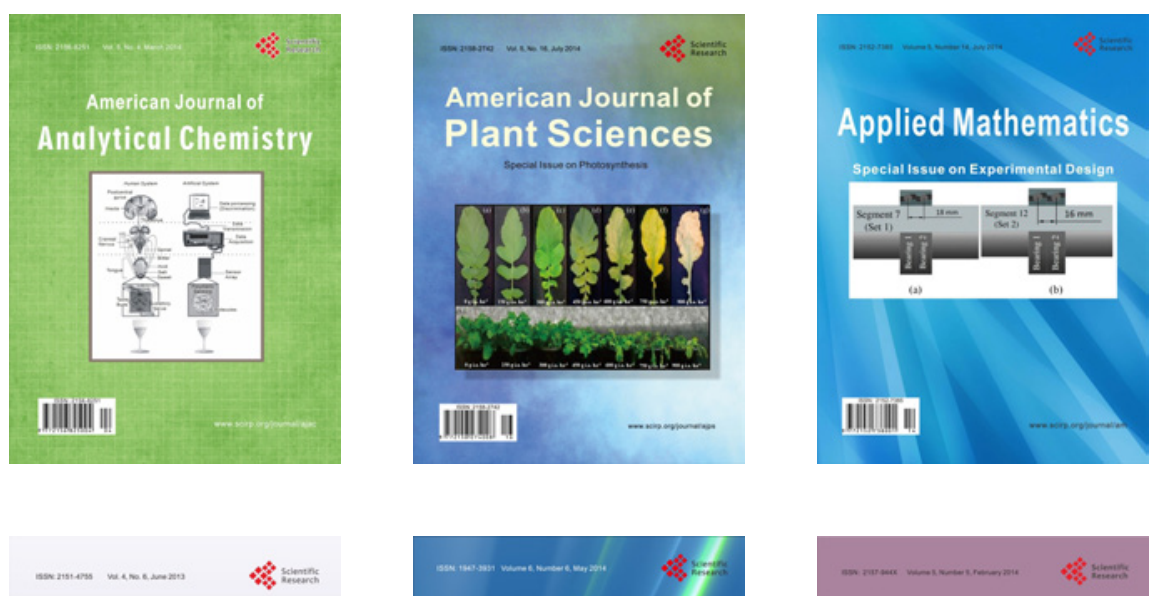

Creative Education
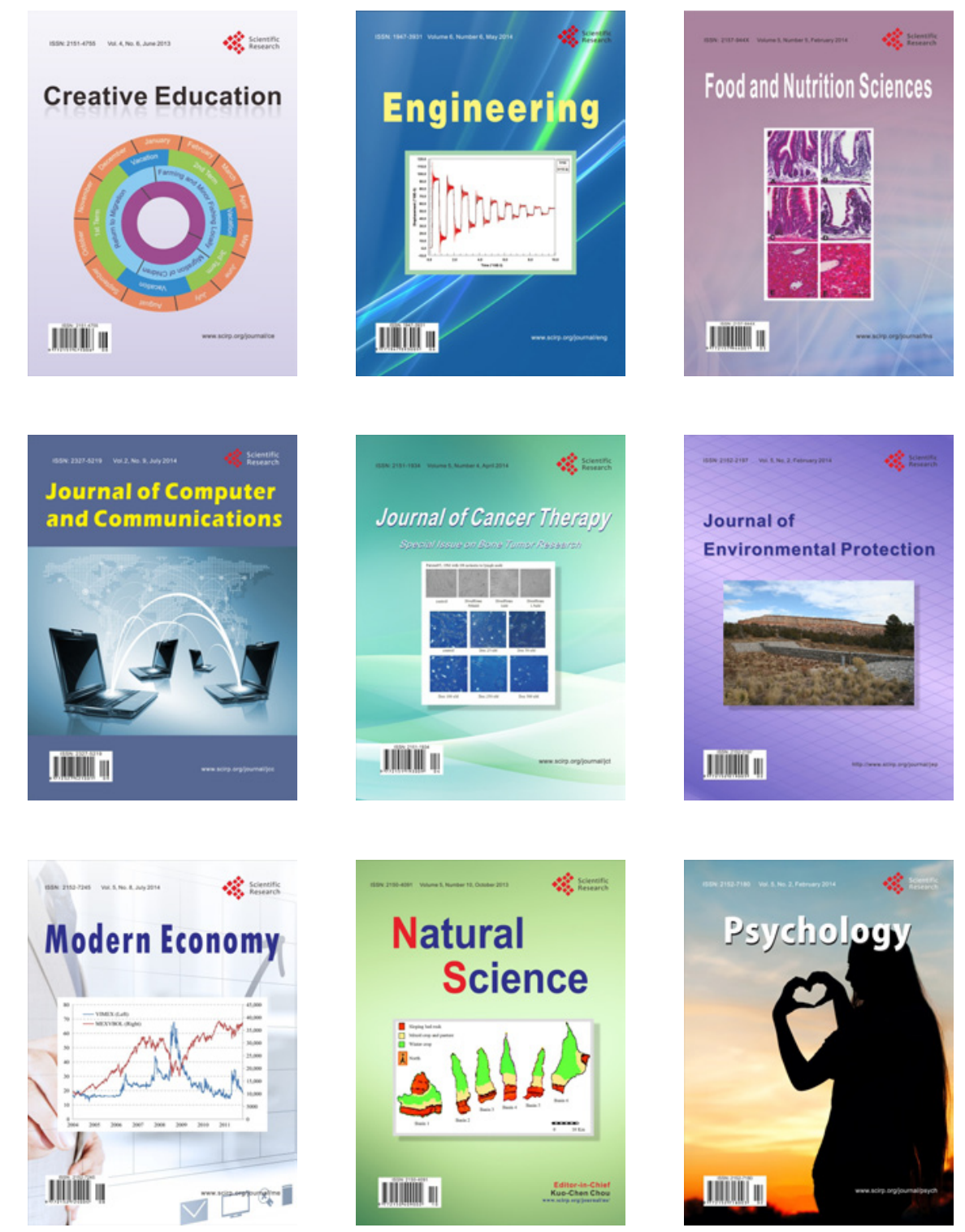